"Social Theory and the Industrial Revolution". In it he examines carefully within the limited time at his disposal very specific questions about eighteenth-century personality formation, laying particular emphasis on religious upbringing and education. His paper breaks much new ground, unlike the other papers, and deserves to be followed up, even if his conclusion is a modest one-that in the study of processes of economic growth "noneconomic factors continue stubbornly and vigorously to assert their existence".

The volume is introduced by an excellent and well balanced introduction by Professors Burns and Saul which sets out neatly a number of points concerning the Hagen debate and admits fairly that despite the fascination of cross-disciplinary argument "a good deal of time was spent during the conference in establishing a mutual discourse rather than in carrying it on".

Asa Briggs

\section{LUNAR INTRODUCTION}

\section{An introduction to the Study of the Moon}

By Z. Kopal. (Astrophysics and Space Science Library, Vol. 4.) Pp. xii + 464. (Dordrecht: D. Reidel Publishing Company, 1966.) 72 D.florins.

THIs new volume, which deals with the Moon's interior, exterior, and environment, is one of the most extensive books on the Moon that I have seen. The twenty-four chapters cover four main topics. The first is the motion of the Moon and dynamics of the Earth-Moon system; the second is a detailed treatment of the internal constitution of the lunar globe; the third topic is the typography of the Moon; and the fourth-and most controversial-is an analysis of the problem of lunar radiation and surface structure. The detailed bibliographical notes accompanying each section forms an invaluable reservoir of research ideas and useful data.

The first part of the book deals with such basic facts as distance, size, mass, and the motion of the Moon in space. There follows a discussion of the problems of the rotation of the Moon and its optical librations. Such details as the motion of the Moon about its centre of gravity and its physical librations make interesting reading. Other consequences of the lunar orbit, such as phases and eclipses, are examined in detail. The last chapter of this section deals with dynamics of the Earth-Moon system, including tidal problems. Kopal concludes that the Moon originated outside of the Earth's orbit. Later the Earth captured it and, through tidal evolution, reduced the orbit to its present characteristics.

The second section, dealing with the Moon's interior, is somewhat less exact. Kopal argues that small but unmistakable departures from hydrostatic equilibrium must exist, because of the Moon's peculiar rotational motion around its centre of gravity. It seems not to be quite spherical. The author next discusses the thermal history of the Moon, including the effects of radioactivity on the temperature of the interior. He goes on to discuss the stresses that may exist inside the Moon, the Moon's chemical composition, the possible convection in the lunar interior, and finally the character of any residual the Moon may possess.

The third section deals with problems of lunar measurement, mapping of the Moon, and finally a detailed description of formations on the lunar surface. Most of the craters, Kopal argues, are of impact origin. Many of the smaller craters result from secondary impacts of the splash material, but he does concede that certain craters, like Ptolemy and Alphonsos and the filled crater Wargentin, must represent some sort of volcanic origin. With reference to dust on the Iunar surface, Kopal points out that the Ranger photograph, which exhibits a vertical differen- tiation on a meter scale, scarcely supports Gold's argument that large reservoirs of fine dust could have drifted to the lowlands from the plateaux. Kopal concludes the section with a discussion of the lunar surface as an impact counter.

The last part starts out with problems of lunar albedo and photometry of scattered moonlight. The author deals next with problems of a lunar temperature and thermal emission of the lunar surface. He next considers the electromagnetic properties of the lunar surface. His discussion of the luminescence of the lunar surface is probably the most controversial. The evidence for such "lunar events", bright areas reported from time to time on the dark side of the Moon, is not well substantiated. On pages 396-7, Kopal shows two photographs taken by himself and Rackham on November 2, 1963, within the same short period of time. He notes that there appears to be a transient red enhancement of the area around Kepler in one of the pictures. In lieu of the possibility that some photographic defects may be responsible, the discussion based on this single picture is not convincing. Future research may indeed bear out Kopal's conclusions concerning the existence of "lunar flares". The case for their existence, however, is far from definite. The last two chapters are essential addenda, to introduce the recent lunar photographs taken by Rangers 7, 8 and 9 .

Although the book has a few weaknesses, as noted here, An Introduction to the Study of the Moon is a valuable contribution to astronomical literature. It is far more than just an introduction. In view of the fact that the author has had substantial contact with still more recent photographs, from the lunar Orbiter series, we may hope for additional analytic volumes concerning the nature of the lunar surface and the origin and history of the various kinds of formations detected on both the near and far sides of the Moon.

D. H. Menzei

\section{FULL OF APPROXIMATIONS}

Methods of Numerical Approximation

Edited by D. C. Handscomb. (Lectures delivered at a Summer School held at Oxford University, September 1965.) Pp. ix +218 . (Oxford, London and New York: Pergamon Press, Ltd., 1966.) 63s. net.

This book, with chapters by J. D. P. Donnelly, L. Fox, D. C. Handscomb and D. F. Mayers of the Oxford University Computing Laboratory and A. R. Curtis and M. J. D. Powell of the Atomic Energy Research Establishment, Harwell, is to be welcomed for the range of material presented, some of it not conveniently available elsewhere. Its subject is "the approximation of functions by means of more elementary functions, regarded as a tool in numerical computation". After an introductory chapter devoted to functional analysis, the book is divided into three sections, "Linear Approximation", "Rational Approximation" and "Miscellaneous".

The first opens with Weierstrass's theorem on polynomial approximations followed by Lagrangian interpolation. Because the book is directed at the user of digital computers, appropriately finite differences are not introduced; instead the authors continue with least squares approximations, showing the advantages of orthogonal functions. This leads naturally to Chebyshev series and various methods for their determination such as economization of power series. The next chapter considers the general problem of best linear approximation. Best uniform (minimax) approximation is shown to be characterized by equi-oscillation of the error and this is followed by a very full description of the important exchange algorithm for constructing such approximations.

The next section introduces continued fractions and interpolation by rational functions. Rational functions 